\title{
Study of antimicrobial resistance for the causative agent of the bovine reproductive system infection by the NGS method
}

\author{
Sergey Zaytsev ${ }^{1}$, Mariya Khizhnyakova ${ }^{1}$, Ekaterina Krasnikova $^{2}$, Olga Larionova $^{1,3}$, \\ Valentina Feodorova ${ }^{1, *}$ \\ ${ }^{1}$ Federal Research Center for Virology and Microbiology, Branch in Saratov, 410028 Saratov, Russia \\ ${ }^{2}$ Federal State Budgetary Educational Institution of Higher Education "Michurinsk State Agrarian \\ University", 393760 Michurinsk, Russia \\ ${ }^{3}$ Federal State Budgetary Educational Institution of Higher Education "Saratov State Agrarian \\ University named after N.I. Vavilov", 410003 Saratov, Russia
}

\begin{abstract}
In the present study, the biomaterial specimen derived from a farm animal with a chronic reproductive system infection was carefully investigated using the Next Generation Sequencing method. The bacterial DNA of the genus Enterobacter was detected. The genetic determinants of resistance to 12 classes of antibiotics were identified in the genome of this microorganism. The data obtained highlight the necessity of strengthening global control over the spread of resistant microorganism strains in agriculture especially in the animal husbandry sector.
\end{abstract}

\section{Introduction}

Infectious diseases of farm animals are an urgent problem in the field of animal husbandry. Infections causing different pathological processes in the reproductive system of animals may reduce the productiveness and productivity of livestock, resulting in significant damage and loss to agribusiness.

According to the reports on the epizootic situation with animal infection diseases in the Russian Federation (https://fsvps.gov.ru/fsvps/iac/rf/reports.html), cases of bacterial infectious diseases associated with reproductive system pathologies are annually registered on the territory of the Russian Federation. In addition to pathogenic microbes, opportunistic microorganisms, including the representatives of the Enterobacteriaceae family and etc., can play an important role as causative agents of infectious diseases in animals [1,2]. The emergence and spread of antibiotic-resistant variants of these types of microorganisms is of serious concern to world health and veterinary specialists. The annual increase in the number of bacterial strains capable of exhibiting resistance to the main classes of antibiotics leads to a fatal decrease in the spectrum of drugs for the effective therapy of infectious diseases in farm animals [3-6].

\footnotetext{
* Corresponding author: feodorovav@mail.ru
} 
Currently, the widespread use of feed antibiotics is considered to be one of the reasons for the geographic expansion of antibiotic-resistant bacterial strains [7].

It is known that the process of bacteria acquiring drug resistance is mediated by their genetic structure [8]. Therefore, a detailed study of bacterial genomes may become the general basis for elucidating the factors of drug resistance of microorganisms and increasing the effectiveness of using antibiotics in the treatment of infectious diseases of productive animals.

Next-generation sequencing (NGS) platforms are modern high-throughput sequencing tools that allow decoding nucleotide sequences, assembling contigs and whole bacterial genomes, as well as identifying genetic determinants of resistance of a specific causative agent of a livestock infectious disease to various classes of antibiotics.

The aim of this work was to study predictive drug resistance in the causative agent of infection of reproductive system in cattle with anamnestic abortions using NGS and bioinformatics approaches.

\section{Materials and Methods}

Total DNA from the bovine specimens was isolated with the DNeasy Blood \& Tissue Kits (QIAGEN, Germany). The isolated DNA was sequenced with the second generation sequencing technique using a high-throughput Illumina HiSeq 2500 sequencer (Illumina inc., USA). The de novo assembly of nucleotide sequences was performed using the SPAdes v. 3.15 .2 (https://cab.spbu.ru/software/spades/). Analysis of contigs for the genetic determinants of antimicrobial resistance was performed using the CARD database (https://card.mcmaster.ca/).

\section{Results and Discussion}

After sequencing the DNA isolated from the sick animal, raw data readings were successfully obtained. Analysis of short sequences with metagenomic approaches made it possible to detect in the clinical sample the presence of DNA which was taxonomically associated with opportunistic microorganisms of the Enterobacteriaceae family. The dominant number of reads ( $>90 \%$ ) belonged to the genus Enterobacter (Fig. 1), indicating the leading role of these microorganisms in the development of the inflammatory process in the animal's reproductive system.

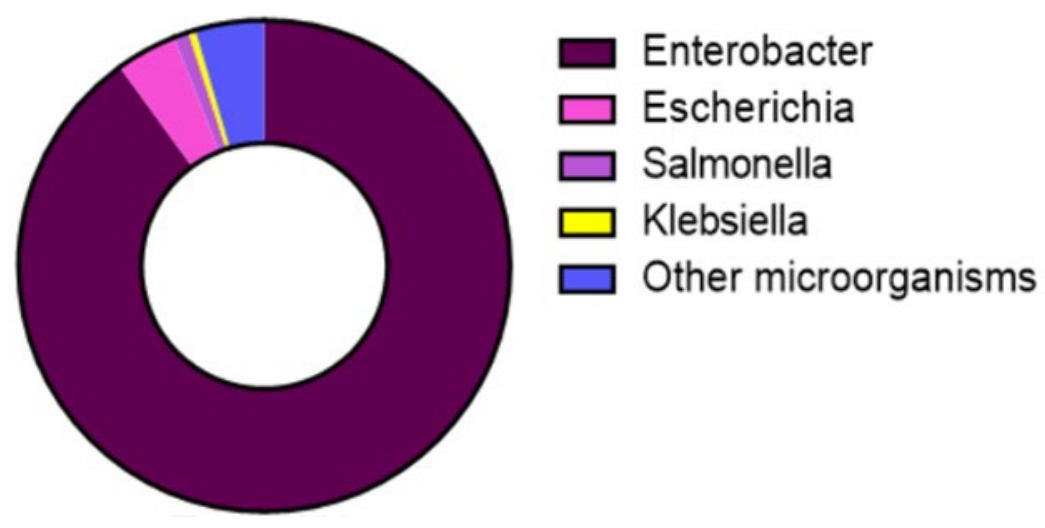

Fig. 1. Result of metagenomic analysis of "raw" reads of DNA isolated from the biomaterial of the animal (cattle) with an reproductive system infection. 
According to the reports $[9,10]$, drug-resistant variants are most common among the Enterobacter genus. Initially, these bacteria were associated mainly with nosocomial infections in humans. However, in recent years, due to the use of molecular genetic research methods, there are more reports on the identification of these microorganisms in specimens of livestock with inflammatory processes of various systems and organs, including the reproductive system [11, 12]. Moreover, the emergence of multi-resistant strains among the Enterobacter genus is of serious concern. According to the global monitoring of the spread of resistant forms of microorganisms CARD, the number of cases in which superbugs were detected among microorganisms of the Enterobacter genus has more than doubled over the past two years (Fig. 2).

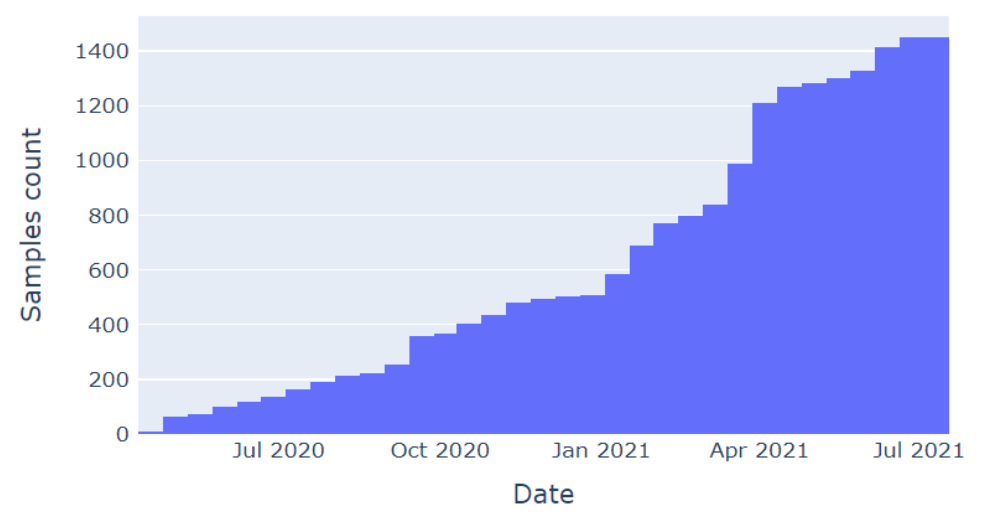

Fig. 2. The number of cases of the detection of multi-resistant strains belonging to the Enterobacter genus in the Russian Federation and abroad in 2020-2021 (according to CARD: Live Visualization Tools).

In order to provide the molecular and genetic characteristics of the microorganism found in this study, the de novo assembly of chromosomal DNA was performed. As a result, the contigs with an average length of about 50,000 bp were obtained. Predictive analysis of the investigated strain with the use of CARD database revealed the presence of nucleotide sequences associated with resistance to antimicrobial drugs of 12 different classes (Fig. 3), such as: carbapenems, aminocoumarins, aminoglycosides, nitroimidazoles, nitrofurans, diaminopyrimidines, macrolides, ansamycins, fluoroquinolones, fosfomycins, and peptide antibiotics. 


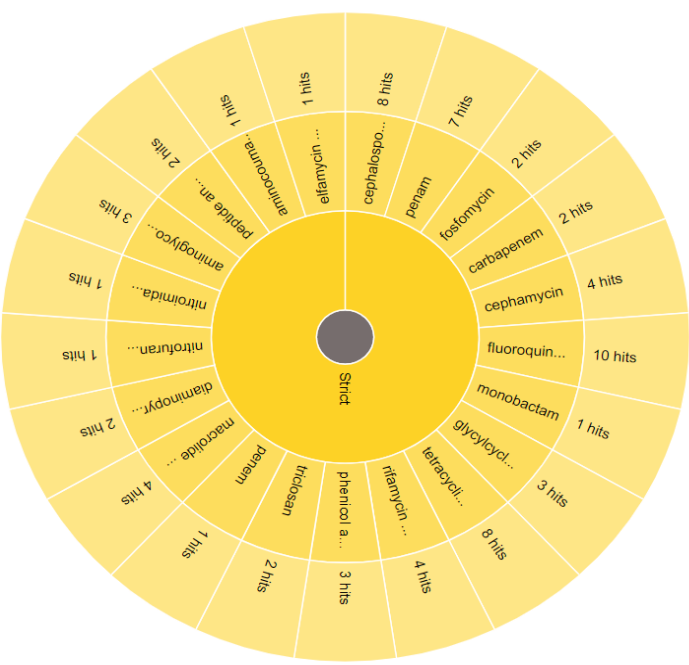

Fig. 3. Classes of antimicrobial drugs which were associated with the genetic determinants of the resistance of the strain derived from the biomaterial of the animal with a reproductive system infection.

The data obtained confirms and supplements the CARD monitoring information on the leading positions of four classes of antibiotics (cephalosporins, carbapenems, fluoroquinolones and aminoglycosides) in the list of drugs. In fact, the acquired resistance of pathogenic and opportunistic microorganisms in Russia is associated with these antibiotics (Fig. 4).

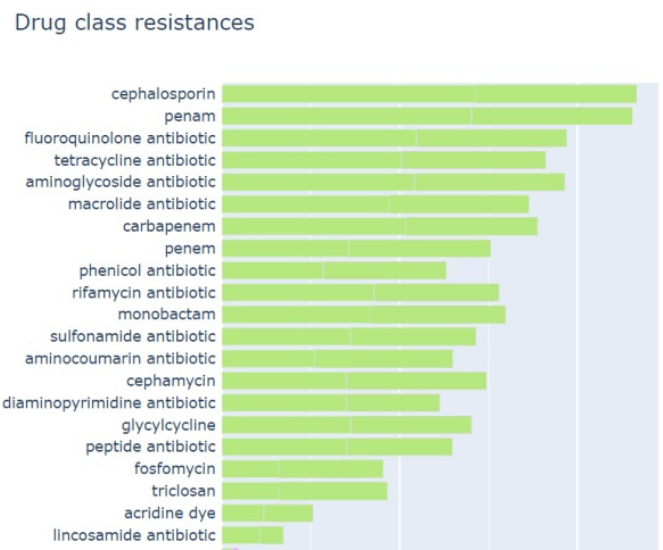

Fig. 4. Classes of antibiotics, the drug resistance to which has been registered in microorganisms on the territory of the Russian Federation in the period of 2020 - 2021 (according to CARD: Live Visualization Tools).

The detection of the Enterobacter genus representative with multiple resistance to antibiotic as the etiological factor for the bovine reproductive tract infection indicates the need to strengthen control over the spread of resistant microorganisms on the territory of the Russian Federation. 


\section{Conclusions}

In the course of this work, the multi-antibiotic-resistant strain belonging to the Enterobacter genus was found in the biomaterial of a farm animal. This microorganism possessed the predictive resistance to the main groups of antibiotics which are used in veterinary practice as part of prophylactic and therapeutic measures. The identification of strains with multiple drug resistance in the farm animal indicates the necessity to strengthen the global control over the spread of antibiotic-resistant microorganisms, which could be a serious threat to animal health.

\section{Acknowledgments}

The study was supported by the Russian Foundation for Basic Research (RFBR), Project No. 19-316-90024.

\section{References}

1. J.A. Gibson, L.E. Eaves, B.M. O'Sullivan, Equine Vet J. 14(2), 122-125 (1982)

2. A. Davin-Regli, J.P. Lavigne, J.M. Pagès, Clin Microbiol Rev. 32(4), e00002-19 (2019)

3. J. O'Neill, Tackling drug-resistant infections globally: final report and recommendations the review on antimicrobial resistance, The review on antimicrobial resistance, 84 (2016)

4. R. Vivs, A.A.T. Barbosa, S.S. Dolabela, S. Jain, Microb Drug Resist 25(6), 890-908 (2019)

5. Antibiotic Resistance Threats in the United States, 2013, CDC, Atlanta, GA, USA (2013)

6. R. Laxminarayan, A. Duse, C. Wattal, A.K.M. Zaidi, H.F.L. Wertheim, N. Sumpradit, E. Vlieghe, G.L. Hara, I.M. Gould, H. Goossens, C. Greko, A.D. So, M. Bigdeli, G. Tomson, W. Woodhouse, E. Ombaka, A.Q. Peralta, F.N. Qamar, F. Mir, S. Kariuki, Z.A. Bhutta, A. Coates, R. Bergstrom, G.D. Wright, E.D. Brown, O. Cars, Lancet Infect. Dis. 13, 1057-1098 (2013)

7. A.V. Zabrovskaya, Farm Animals 1, 78-83 (2013)

8. A.H. Holmes, L.S.P. Moore, A. Sundsfjord, M. Steinbakk, S. Regmi, A. Karkey, P.J. Guerin, L.J.V. Piddock, Lancet 387, 176-187 (2016)

9. M.K. Annavajhala, A. Gomez-Simmonds, A.C. Uhlemann, Front. Microbiol 10, 44 (2019)

10. R.P. Chakrabarty, M. Sultana, S. Shehreen, S. Akter, M.A. Hossain, AMB Express. 6(1), 126 (2016)

11. Z. Wang, L. Duan, F. Liu, Y. Hu, C. Leng, Y. Kan, L. Yao, H. Shi, BMC Veterinary Research 16, 1 (2020)

12. W. Shan-Shan, S. Yun-Jia, C. Xing-Yang, W. Cheng-Wei, G.U. Shan-Shan, Y. Xin, X. Shuang, G.E. Jun-Wei, C. Hong-Yan, Chin. Vet. Sci. 47, 768- 772 (2017) 